\title{
PRECISE POSITIONING OF PIEZO-ACTUATED STAGES USING HYSTERESIS-OBSERVER BASED CONTROL
}

\author{
Chih-Jer Lin and Sheng-Ren Yang \\ Department of Mechanical and Automation Engineering, Da-yeh University, \\ 112 Shan-Jeau Rd., Da-Tsuen, Chang-Hwa Country, Taiwan (R.O.C.), 51505. \\ Email: cjlin414@mail.dvu.edu.tw
}

\begin{abstract}
The piezo-actuated stages are composed of the piezo-electric actuator and the positioning mechanism. The positioning accuracy of the piezo-actuated stage is limited due to hysteretic nonlinearity of the PEA and friction behaviour of the positioning mechanism. To compensate this nonlinearity of piezoelectric actuator, a PI feedback control associated with feedforward compensating based on the hysteresis observer is proposed in this paper. To verify the consistency of the proposed method, the experiments are implemented by real-time control to be compared with the numerical simulation. Copyright $(\mathrm{C} 2005$ IFAC
\end{abstract}

Keywords: Modelling, Quadratic programming, Parameter estimation, Observers, Realtime.

\section{INTRODUCTION}

Piezoelectric actuators (PEAs) are effective elements in ultra-high-precision positioning applications, such as scanning probe microscopy (SPM), scanning tunnelling microscopy (STM), optical alignments, diamond turning machines, active vibration control of rotor bearing systems, and tracking and focusing of a hard disk driver (Uchino, 1997); PEAs are used to meet the requirements of nanometer resolution in displacement, high stiffness, and rapid response. However, its main disadvantageous characteristic is the nonlinearity of hysteresis between the displacement and the electric field strength; other causes for loss in precision of positioning are drift due to creep effects (Croft, et al., 2000) and temperature effects. The hysteresis is not a differentiable and one-to-one nonlinear mapping but nonlinearity with local memory, so that it causes positioning errors which critically limit the operating speed and precision of PEAs. Nonlinear hysteresis effects can be corrected using charge control; however, it may lead to drift and saturation problems as well as reduce the operating range greatly. The other solution is to establish the models of the hysteresis nonlinearity and improve the precision with inverse model feed-forward compensation. Many studies have modelled the nonlinearity of hysteresis of PEAs, such as the Preisach model (Ge and Jouaneh, 1997), the Duhem model (Stepanenko and $\mathrm{Su}, 1998$ ), the Maxwell slip model (Goldfarb and Celanovic, 1997), the constant phase lag approximation, or the Bouc-Wen model (Xueliang, et al., 1997). However, the loss in precision due to the creep effects and temperature effects cannot be improved only by these feedforward compensating methods and the most direct method is to incorporate the feedforward control with the feedback compensation. Based on the Preisach model, Ge and Jouaneh developed a controller consisting of a feedforward tracking part and a PID feedback part (Ge and Jouaneh, 1997). Croft and Devasia applied the polynomial equations to describe the inverse model of the hysteresis and designed a controller with PD-type feedback to achieve the tracking control (Croft and Devasia, 1998). Recently, Hwang et al. applied the novel neural-network model which includes two different nonlinear gains according to change rate of input signal and a linear dynamic system to learn the piezomechanism.

Most of the above studies are focused on tracking cyclic reference signals of the impact-driven mechanisms with the symmetry hysteresis, however applications of scanning-type devices such as scanning electron microscope (SEM) and atomic force microscope (AFM) require positioning of the probe or the stage in the sub-micro range to focus or scan specimens. Therefore, in this paper, positioning control of the stage with the embedded PEA is studied. Especially, the studied piezo-actuated stage 
has the non-symmetric hysteresis, because the PEA is embedded and constrained by the positioning mechanism. For the modelling of non-symmetric hysteresis, the polynomial model and the Preisach model are usually applied to compensate the nonlinearity. However, the polynomial model may cause errors if the amplitude of the reference input is not the same as the original. The traditional Preisach model is based on a collection of measured input/output datasets including samples from the major ascending curve and many first order reversal curves. However, the disadvantage is that it does not accurately describe the minor loop hysteresis behaviour. The modified Preisach model can predict the minor loop behaviour, but it increases the complexity and the size of the stored datasets due to the additional measured data of second order reversal curves. These two methods establish the nonlinear relations between the input and output based on the measured input/output datasets, but the dynamic of the PEA does not be formulated. The Duhem's model and the Bouc-Wen model used nonlinear differential equations to describe the hysteresis of a PEA, whose mathematical formulas for the hysteresis model can describe the dynamics of the PEA. These two methods are more efficient to implement than modelling of the Preisach model or the polynomial model; however, their disadvantage is that they cannot formulate the PEA model with the non-symmetric hysteresis. To solve the above problems, in this paper, a PI feedback control associated with the feedforward compensating based on the hysteresis observer is proposed. A hysteresis observer based on the Bouc-Wen model is proposed to describe hysteresis nonlinearity of the PEA and the dynamic of the positioning mechanism is identified by the impulse response method; on the other hand, to compensate the modelling uncertainties due to the non-symmetric hysteresis and external disturbances, the PI feedback control is incorporated to the proposed controller.

\section{MAIN RESULT}

\subsection{The dynamic equation of the system}

In this section, the modelling of the piezoactuated positioning stage will be derived first and then the observer-based feedforward control incorporated the PI feedback control is proposed. The studied Piezo-actuated positioning stage to achieve the precision positioning is made by Piezomechanik GmbH and it is described in Fig. 1. The model is composed of the positioning mechanism (PM) and the piezo electric actuator (PEA); the PM can be modelled as a mass-springdamper mechanic system by a linear time-invariant second order differential equation, and the PEA can be regarded as a force generator which generates force due to the applied voltage. The dynamic equation of the piezo-actuated positioning stage can be detailed in Fig. 2 and formulated as follows:

$m \ddot{x}+b \dot{x}+k x=u_{h}$ where $u_{h}$ represents the force generated by the PEA with hysteresis and $x$ is the displacement of the stage; $\dot{x}, \ddot{x}$ are the first and second order derivatives of $x$ with respect to time $t$, respectively; the parameter $m$ is the mass, $k$ is the stiffness factor and $b$ is the viscous friction coefficient of the PM.

\subsection{The modelling of the Piezo-actuated stage}

To identify the parameters $m, b, k$ of the positioning mechanism, the impulse response method is applied to find the natural frequency and the damping ratio by the impact hammer (Kistler, 9722A500), the laser positioning sensor (National, LM300 ANL3531C2) and the dynamic signal analyzer (Addlink: siglab 20-42); the testing scheme is described in Fig. 3. From the results of the impulse response, the damping ratio and natural frequency can be obtained as follows.

$$
\begin{aligned}
& 2 \pi \xi=\ln \left(\frac{-0.0364695}{-0.0196374}\right) \Rightarrow \xi=0.098 \\
& f=\frac{1}{0.00320313-0.00179688} \Rightarrow \omega_{n}=4465(\mathrm{rad} / \mathrm{s})
\end{aligned}
$$

The nominal mass of the positioning stage is $m=0.148 \mathrm{~kg}$ and then the stiffness factor and the damping coefficient can be determined from the following equations:

$$
\begin{aligned}
& b / m=2 \xi \omega_{n} \\
& k / m=\omega_{n}{ }^{2} \\
& \text {, and } b=129.5 \mathrm{~N}-\mathrm{s} / \mathrm{m}, k=2.95 \times 10^{6} \mathrm{~N} / \mathrm{m} .
\end{aligned}
$$

From the above experiment, the modelling of PM can be obtained; however, this model is not yet complete, because the relation between actuating forces and applied voltages has not yet been modelled. For a PEA, the relation between actuating force and applied voltage is not linear but a nonlinear hysteresis model. Therefore, it can be measured by the experimental loops for the relation between the displacements and applied voltages. The nominal displacement range of the PEA is about $-9 \mu m \sim+30 \mu m$ when the applied voltage range is $-30 \mathrm{~V} \sim+100 \mathrm{~V}$. In the experiments, the laser interferometer (HP-5529A) with 10nm resolution and a $3 \mathrm{MHz}$ maximum data update rate measures the actual displacement of the Piezo-actuated stage, and the measured hysteretic loop and the simulation result by the Preisach model are also described in Fig. 4 ; where the major loop is obtained by the applied voltage of the $1 \mathrm{~Hz}$ triangle waveform with the amplitude of $100(\mathrm{~V})$; the first and second minor loops are obtained by the $1 \mathrm{~Hz}$ triangle waveforms with the amplitudes of $60(\mathrm{~V})$ and $30(\mathrm{~V})$. The Preisach model can be used to describe the hysteresis as in Fig. 4; however, it may cause errors due to the unmatched reference input. To solve this problem, the dynamic of hysteresis should be established by the nonlinear differential equations. In this paper, the Bouc-Wen model is applied to describe the nonlinearity of the PEA; the total modelling of the Piezo-actuated stage can be described as follows. 


$$
\left\{\begin{array}{c}
m \ddot{x}+b \dot{x}+k x=u_{h}=k(d u-h)+\rho \\
\dot{h}=\alpha d \dot{u}-\beta|\dot{u}| h-\gamma \dot{u}|h|
\end{array}\right.
$$

where $h$ represents the hysteretic nonlinear term and $x$ is the displacement of the stage; $\dot{x}, \ddot{x}$ and $\dot{h}$ are the derivatives of $x$ and $h$ with respect to time $t$, respectively; $u$ is the applied voltage and $d$ is the ratio of the displacement vs. the applied voltage; $\rho=k x_{0}$ and $x_{0}$ is the initial displacement as $u=0$; $\alpha, \beta, \gamma$ are the parameters to determine the hysteretic loop's magnitude and shape; the parameters $m, b, k$ are the mass, the stiffness factor and the damper coefficient of the PM. The Bouc-Wen model can be used to describe the symmetric hysteresis nonlinear relation by its parameters' identification; the modified quadratic programming is proposed to identify the optimal set of the parameters $\alpha, \beta, \gamma$ with respect to the root-mean-square error between the actural system and the Bouc-Wen model as the following.

$$
\begin{gathered}
\text { Minimize } E_{\alpha, \beta, r}=\sqrt{\frac{\sum_{i=1}^{N}\left[x_{h}(i)-x_{B W}(i)\right]^{2}}{N}} \\
\text { subject to }\left\{\begin{array}{r}
m \ddot{x}_{B W}+b \dot{x}_{B W}+k x_{B W}=u_{h}=k(d u-h)+\rho \\
\dot{h}=\alpha d \dot{u}-\beta|\dot{u}| h-\gamma \dot{u}|h|
\end{array}\right. \\
{\left[\begin{array}{l}
L B_{1} \\
L B_{2} \\
L B_{3}
\end{array}\right] \leq\left[\begin{array}{l}
\alpha \\
\beta \\
\gamma
\end{array}\right] \leq\left[\begin{array}{l}
U B_{1} \\
U B_{2} \\
U B_{3}
\end{array}\right]}
\end{gathered}
$$

, where $i$ is the sampling index and $N$ is the total number of samples, $x_{h}$ is the actual displacement with respect to the applied voltage $u$, the cost function of this optimal problem is defined as the root-mean-square error between the Bouc-Wen model and the actual system, $L B_{i}, U B_{i}$ are the lower and upper bounds of search ranges for $\alpha, \beta, \gamma$. If $\mathrm{n}$ is the length of search sets, a simplex in n-dimensional space is characterized by the $n+1$ distinct vectors which are its vertices; at each step of the search, a new point in or near the current simplex is generated. The function value at the new point is compared with the function's values at the vertices of the simplex and, usually, one of the vertices is replaced by the new point, giving a new simplex. This step is repeated until the diameter of the simplex is less than the specified tolerance and these optimal parameters are described in Table 1 with $E_{r m s}=0.1161$. The approximating relation obtained by the Bouc-Wen model with the parameters is detailed in Fig. 5; from Fig. 5, the Bouc-Wen model can not describe the non-symmetric hysteresis exactly. However, the difference between the actual hysteresis and the Bouc-Wen model can be considered as the modelling uncertainties and compensated by the PI feedback. To compensate the nonlinearity due to the hysteresis of the PEA, it is necessary to design a feedforward controller to determine the inverse-hysteretic relation between the applied voltage and the displacement. In this paper, the concept of hysteresis observer is proposed to obtain this inverse-hysteresis relation as the following.

\subsection{The feedforward compensating and the feedback compensating based on the hysteresis observer}

Because the hysteresis $h$ in Equation (5) cannot be measured by the sensor, the main propose of the hysteresis observer is to estimate $h$. From the above identification of the Piezo-actuated stage using the Bouc-Wen model, if the modelling error can be compensated by the feedback control and then the model can be considered as the nominal model of the system. Therefore, the Equation (2) can also be arranged in the matrix form as follows.

$\left\{\begin{array}{l}\dot{X}=A X+B u+\left|u_{2}\right| N X+u_{2} I|X| \\ y=C X\end{array}\right.$
where
$A=\left[\begin{array}{ccc}0 & 1 & 0 \\ -\frac{k}{m} & -\frac{b}{m} & -\frac{k}{m} \\ 0 & 0 & 0\end{array}\right], B=\left[\begin{array}{cc}0 & 0 \\ \frac{k d}{m} & 0 \\ 0 & \alpha d\end{array}\right], N=\left[\begin{array}{ccc}0 & 0 & 0 \\ 0 & 0 & 0 \\ 0 & 0 & -\beta\end{array}\right]$,
$I=\left[\begin{array}{lll}0 & 0 & 0 \\ 0 & 0 & 0 \\ 0 & 0 & \gamma\end{array}\right], \quad C=\left[\begin{array}{lll}0 & 0 & 1\end{array}\right], \quad X=\left[x_{1}, x_{2}, x_{3}\right]^{T}$ where $x_{1}=x, x_{2}=\dot{x}_{1}=\dot{x}, x_{3}=\hat{h}$, and $u=\left[u_{1}, u_{2}\right]^{T}$ where $u_{1}=u, u_{2}=\dot{u}$; the output of the hysteresis observer is the estimated hysteresis term $\hat{h}$.

If the hysteresis term of the system without feedforward compensating is considered as $h$ and the actual relationship between the applied voltage $u$ and the output displacement $x$ can be described as follows.

$x(t)=G(u(t), h(t))=d \cdot u(t)-h(t)$

, where $d$ is the ratio of the ideal displacement with respect to the applied voltage; however, the actual displacement is $x(t)$ if the hysteresis is taken into consideration. As a result, if the hysteresis term $h(t)$ can be estimated by the hysteresis observer and then the feedforward compensating based on the hysteresis observer can be designed as follows.

$u_{\text {feedforward }}(t)=f(u(t), \hat{h}(t))=u(t)+\frac{\hat{h}(t)}{d}$

, where $u(t)$ is the original reference and $h(t)$ is the hysteresis state obtained by the hysteresis observer. The feed-forward controller can be obtained by combining the reference input and the hysteresis variable of the observer in Fig. 6; however, the modelling uncertainties and the external disturbance usually exist so that the feedback controller is needed to enhance the robustness of the system and improve the tracking performance and it is described in Figure 7; the feedforward controller in Equation (6) is combined to the PI feedback controller as follows.

$u(t)=K_{P} e(t)+K_{I} \int e(t) d t+\frac{\hat{h}(t)}{d}$

, where $e(t)=x_{d}(t)-x(t)$ represents the positioning error of the system with the feedforward compensating of the inverse hysteresis. 


\section{SIMULATION AND EXPERIMENTAL RESULTS}

In this section, the tracking and positioning performance of the proposed method is evaluated through the simulation cases and compared from the Preisach approach; finally, the experiments of tracking and positioning are designed to show if the proposed method is valid.

\subsection{The simulation case studies}

To check the robustness of the two methods when the amplitudes of the input signals are different from the modelling data, only the output response with respect to the input triangle wave with the amplitude of $100 \mathrm{~V}$ in $1 \mathrm{~Hz}$ frequency is provided for the modelling data; however, the tracking reference with the amplitude of $9 \mu \mathrm{m}$ in the first cycle and $18 \mu \mathrm{m}$ in the second and $29 \mu \mathrm{m}$ in the third is used to check the robustness of the two methods. The simulation results of the feedforward compensating based on the Preisach model and the proposed method are described in Fig. 7 and 8; from the results, the Preisach model is not robust to the different reference input, this is due to the input-output relations of the first two cycles have not been established. However, the same feed-forward control of the proposed method in the section 2 is used for the input reference with the varied amplitudes; the simulation results show that the proposed method has the better robust property than the Preisach approach; this is because the proposed modelling method contains the dynamics of the positioning stage. To obtain the better tracking performance, the feedback controller is combined to the feedforward controller as the same in Fig. 7 and the parameters of the feedback controller are designed as the following: $K_{P}=2.8, K_{I}=280$; the simulation results of positioning task is shown in Fig. 10 and it achieves the excellent positioning when the PI feedback controller combined with the proposed method is applied to the Piezo-actuated stage.

\subsection{The experimental setup}

The laser interferometer (HP-5529A) can measure the displacement of the Piezo-actuated stage for modelling; however, even though it has a $10 \mathrm{~nm}$ resolution, it is not suitable as the feedback sensor of the real-time control due to the cost and practicability. To implement the proposed method on the Piezoactuated stage, we equip the linear encoder and the linear scale with $0.1 \mu m$ resolution (Mercury 2000, made by MicroE systems in U.S.A.) on it to be the positioning sensor and the setup is detailed in Fig. 2. Besides, to achieve the real-time control of the PI feedback control associated with the feedforward compensating of the inverse hysteresis, the DS1104 PPC control board (with 4 16-bit AD, 4 12-bit AD, 8 16-bit DA, 2 Encode, embedded Power PC $603 \mathrm{e} / 250 \mathrm{MHz}$ with $8 \mathrm{MB}$ Flash RAM and $32 \mathrm{MB}$ SDRAM, made by dSPACE in Germany) is used as the real-time controller and the control scheme is described in Fig. 11. In the closed-loop experiments, the real-time control is performed by the architecture detailed in Fig. 12; the feedforward controllers of the inverse hysteresis based on the Preisach model and the proposed method are programmed in Matlab simulink; the positioning signal provided by the linear encoder are decoded by the block DS1104ENC_POS_C1 and the analog signal is outputed by the block DS1104DAC_C2. The output of DS1104DAC_C2 is connected to the input of DS1104ADC_C 5 and the analog signal is transferred to the digital signal by the block DS1104ADC_C5 and the positioning error is computed by comparing the reference input with the output of DS1104ADC_C5; the PI feedback control combined with the feedforward control produces the contol voltage and it is transferred into the analog signal by the block DS1104DAC_C1, and then the output signal is amplified by the voltage amplifier (Trek 601C) to actuate the piezo. Finally, the Matlab simulink program is complied and downloaded into the DS1104 PPC for implementing the real-time task.

\subsection{The experimental results}

To compare the closed-loop responses of the feedback control combined with the feedforward compensating based on the Preisach model and the proposed method, the same tracking task as the simulation case is achieved first. The experimental result of the Preisach method is described in Fig. 12 and the result of the proposed one is described in Fig. 13. From the results of the Preisach, even though the feedback control is combined to it, there also exist some error in the peaks of the first two cycles; however, the tracking error is reduced much smaller than the former in Fig. 8. From the results of the proposed method in Fig. 13, it is clear that the tracking error is improved and is smaller than the Preisach method and the former with only the proposed feedforward control in Fig. 9. Second, to validate the positioning performance of the proposed method, the positioning task described in Fig. 14 and 15 are performed by the proposed method. The task with $0.5 \mu \mathrm{m}$ step is used to test the positioning performance for the short distance. The experimental results show that the proposed method is corresponding to the simulation result and is valid to the positioning task.

\section{CONCLUSION}

The Preisach model is based on a collection of measured input/output datasets including samples from the major curve and minor curves; however, it has worse robustness when the amplitudes of the input signals are different from the modelling data. The proposed observer-based feedforward method has better robustness for the different inputs, because the system is modelled by the dynamics of the positioning stage with hysteresis nonlinearity. To ensure the implementation of the proposed method, the feedback control cooperated with the proposed 
feedforward compensating is achieved by the realtime control; the experimental results show that the proposed is valid and they correspond to the simulation results.

Table 1 The modelling parameters

\begin{tabular}{|c|c|c|l|}
\hline$m$ & $0.148 \mathrm{~kg}$ & $d$ & $1.59 \times 10^{-8}$ \\
\hline$b$ & $129.5 \mathrm{~N}-\mathrm{s} / \mathrm{m}$ & $\alpha$ & 0.385 \\
\hline$k$ & $2.95 \times 10^{6} \mathrm{~N} / \mathrm{m}$ & $\beta$ & 0.0235 \\
\hline$\rho$ & $0.05 \mathrm{~N}$ & $\gamma$ & 0.0495 \\
\hline
\end{tabular}

\section{ACKNOWLEDGE}

The work was supported by the National Science Council, Taiwan, under the grant No. NSC 92-2218E-212-005.

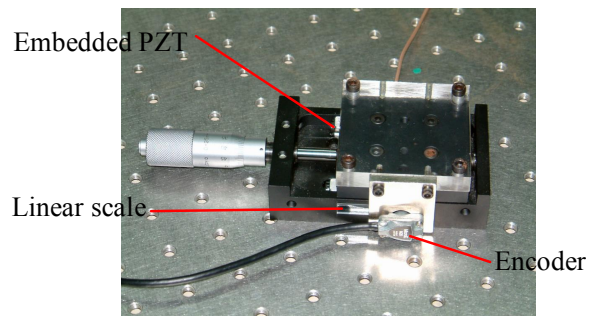

Fig.1. The piezo-actuated stage system with the linear scale and encoder.

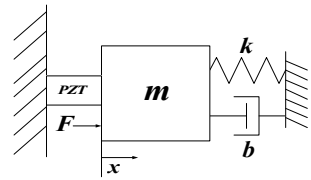

Fig.2. The modelling scheme of the piezo-actuated positioning stage.

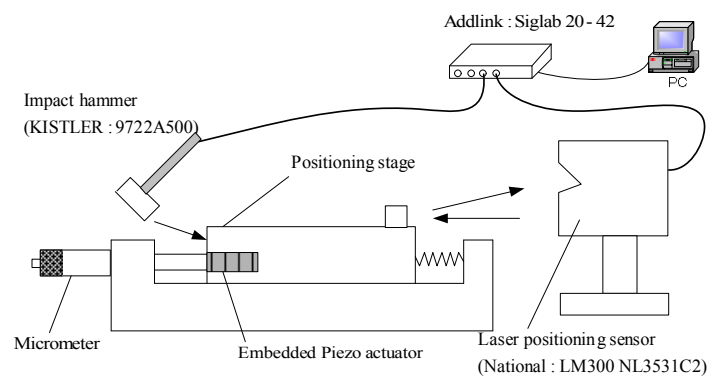

Fig.3. The testing scheme for obtaining the modelling parameters of the PM.

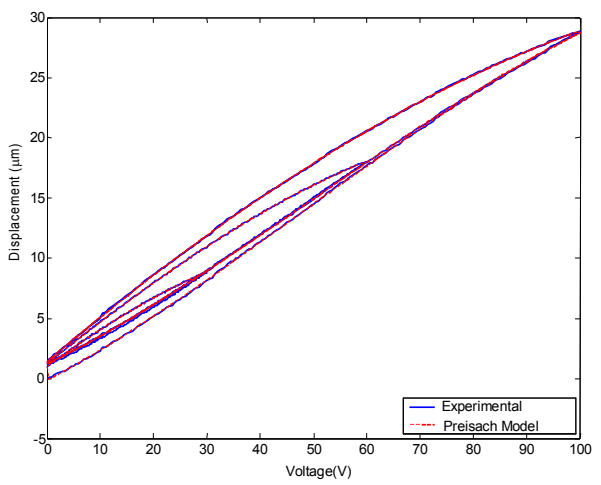

Fig.4. The measured hysteretic loop and the simulation result by the Preisach model.

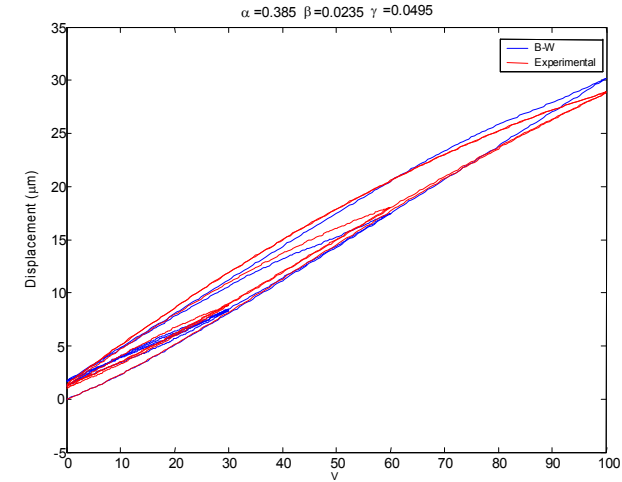

Fig.5. The measured hysteretic loop and the simulation result by the Bouc-Wen model

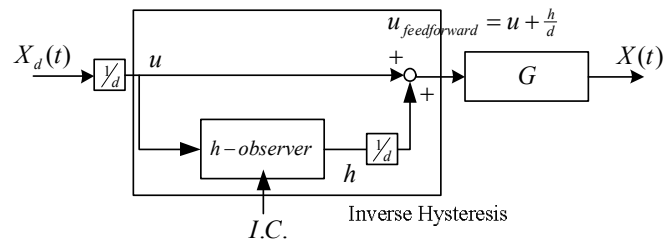

Fig.6. The proposed feedforward compensating method of inverse hysteresis.

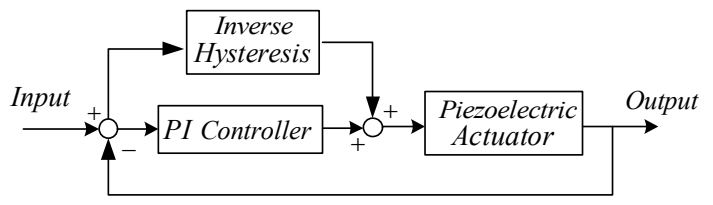

Fig.7. The proposed feedforward compensating incorporated with PI feedback control scheme.

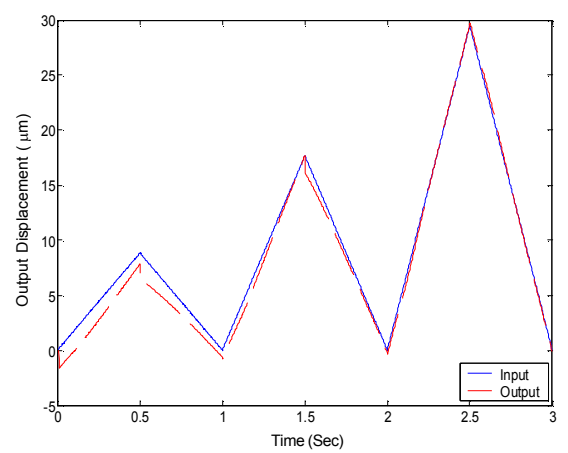

Fig.8. The simulation result by feedforward compensating based on the Preisach model.

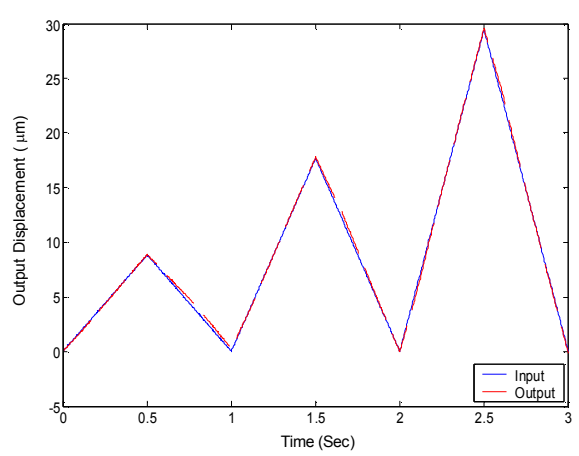

Fig.9. The simulation result by feedforward compensating based on the proposed method. 


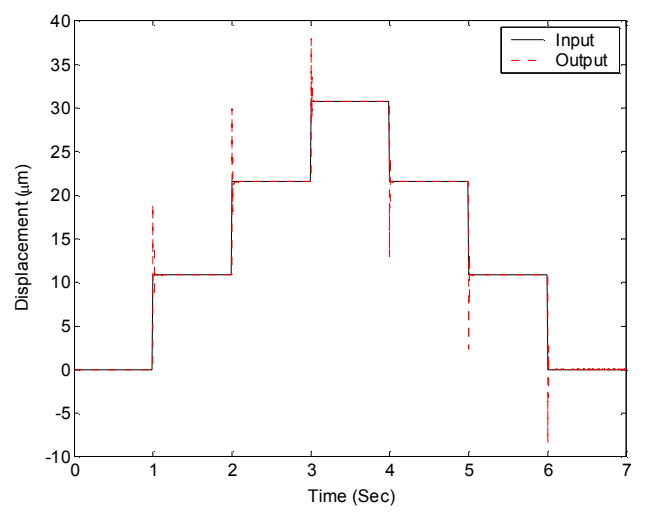

Fig.10. The simulation result of $10 \mu \mathrm{m}$ positioning control by the proposed method.

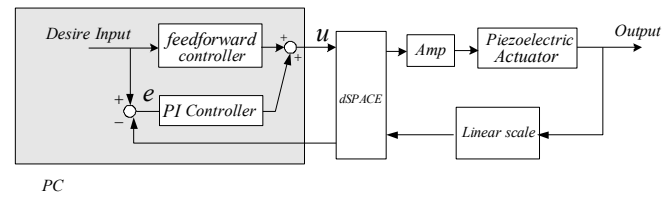

Fig.11. The real-time control architecture.

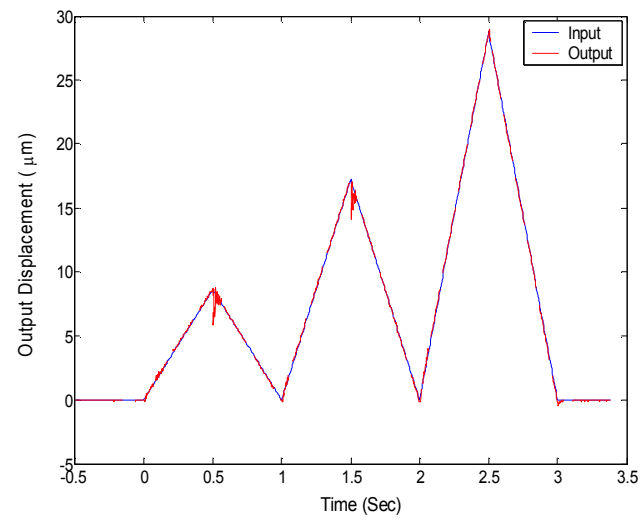

Fig. 12. The experimental result of tracking control by the Preisach method.

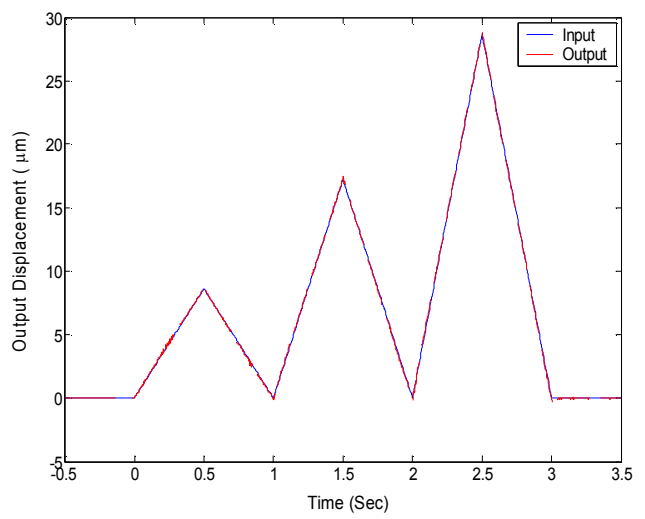

Fig. 13. The experimental result of tracking control by the proposed method.

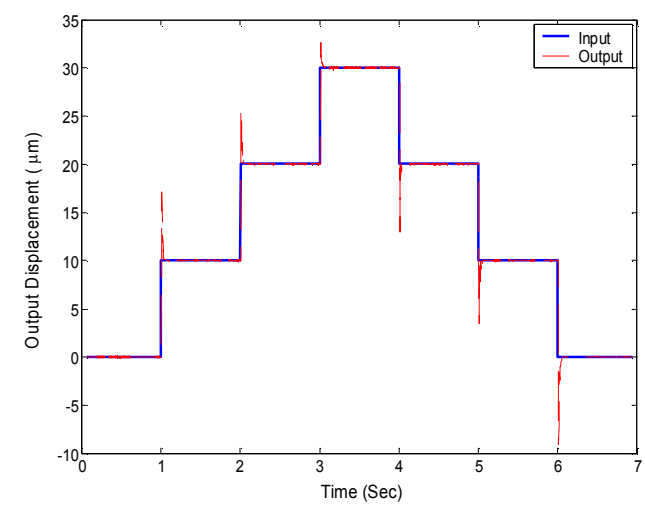

Fig.14. The experimental result of $10 \mu \mathrm{m}$ positioning control by the proposed method.

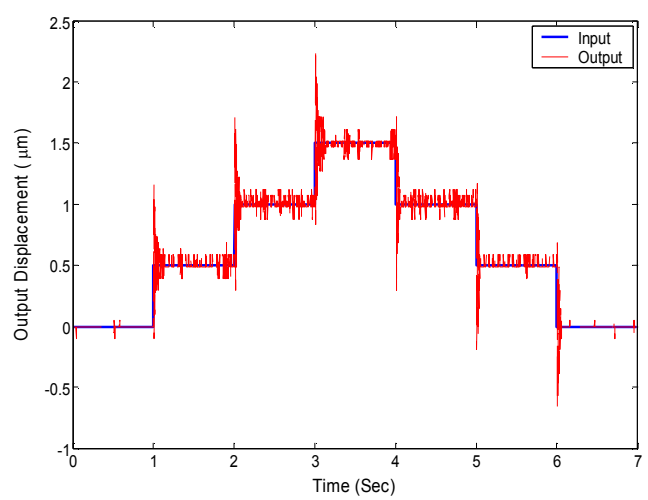

Fig.15. The experimental result of $0.5 \mu \mathrm{m}$ positioning control by the proposed method.

\section{REFERENCES}

Uchino, K. (1997). Piezoelectric Actuator and Ultrasonic Motors, Kluwer Academic Publishers, Norwell MA.

Croft, D., Shedd, G., and Devasia, S. (2000). Proc. of American Control Conference, Chicago, Illinois, 2123-2128.

Ge, P. and Jouaneh, M. (1997). Generalized Preisach Model for Hysteresis Nonlinearity of Piezoceramic Actuator. Precision Engineering, 20, 99-111.

Stepanenko, Y. and Su, C. Y. (1998). Intelligent Control of Piezoelectric Actuators. Proc. of $37^{\text {th }}$ IEEE Conference on Decision and Control, Tampa, FL, 4234-4239.

Zhang, X. L., Huang, Y., Liu, Y. C., Wang, X. Y., and Feng, G. (1997). Proc. of IEEE International Conference on Intelligent Processing Systems, BeiJing, China, 602-605.

Goldfarb, M. and Celanovic, N. (1997). A Lumped Parameter Electromechanical Model for Describing the Nonlinear Behaviour of Piezoelectric Actuators. J. Dynamic Systems, Measurement, and Control, Transactions of the ASME, 119, 478-485.

Croft, D., \& Devasia, S. (1998). Hysteresis and Vibration Compensation for Piezoactuators, Journal of Guidance, Control and Dynamics, 710-717. 